\title{
ON THE SIGNED (TOTAL) $k$-INDEPENDENCE NUMBER IN GRAPHS
}

\author{
Abdollah KhodKar \\ Department of Mathematics \\ University of West Georgia \\ Carrollton, GA 30118, USA \\ e-mail: akhodkar@westga.edu \\ BABAK SAMADi \\ Department of Mathematics \\ Arak University \\ Arak, Iran \\ e-mail: b-samadi@araku.ac.ir
}

AND

\author{
Lutz VolKmanN \\ Lehrstuhl II für Mathematik \\ RWTH Aachen University \\ 52056 Aachen, Germany
}

e-mail: volkm@math2.rwth-aachen.de

\begin{abstract}
Let $G$ be a graph. A function $f: V(G) \rightarrow\{-1,1\}$ is a signed $k$ independence function if the sum of its function values over any closed neighborhood is at most $k-1$, where $k \geq 2$. The signed $k$-independence number of $G$ is the maximum weight of a signed $k$-independence function of $G$. Similarly, the signed total $k$-independence number of $G$ is the maximum weight of a signed total $k$-independence function of $G$. In this paper, we present new bounds on these two parameters which improve some existing bounds. Keywords: domination in graphs, signed $k$-independence, limited packing, tuple domination.
\end{abstract}

2010 Mathematics Subject Classification: 05C69. 


\section{REFERENCES}

[1] M. Chellali, O. Favaron, A. Hansberg and L. Volkmann, $k$-domination and $k$ independence in graphs: A survey, Graphs Combin. 28 (2012) 1-55. doi:10.1007/s00373-011-1040-3

[2] R. Gallant, G. Gunther, B.L. Hartnell and D.F. Rall, Limited packing in graphs, Discrete Appl. Math. 158 (2010) 1357-1364. doi:10.1016/j.dam.2009.04.014

[3] A.N. Ghameshlou, A. Khodkar and S.M. Sheikholeslami, On the signed bad numbers of graphs, Bulletin of the ICA 67 (2013) 81-93.

[4] F. Harary and T.W. Haynes, Double domination in graphs, Ars Combin. 55 (2000) 201-213.

[5] V. Kulli, On n-total domination number in graphs, in: Graph Theory, Combinatorics, Algorithms and Applications, SIAM (Philadelphia, 1991) 319-324.

[6] D.A. Mojdeh, B. Samadi and S.M. Hosseini Moghaddam, Limited packing vs tuple domination in graphs, Ars Combin., to appear.

[7] D.A. Mojdeh, B. Samadi and S.M. Hosseini Moghaddam, Total limited packing in graphs, submitted.

[8] L. Volkmann, Signed k-independence in graphs, Cent. Eur. J. Math. 12 (2014) 517528 . doi:10.2478/s11533-013-0357-y

[9] L. Volkmann, Signed total k-independence number in graphs, Util. Math., to appear.

[10] H.C. Wang and E.F. Shan, Signed total 2-independence in graphs, Util. Math. 74 (2007) 199-206.

[11] H.C. Wang, J. Tong and L. Volkmann, A note on signed total 2-independence in graphs, Util. Math. 85 (2011) 213-223.

[12] D.B. West, Introduction to Graph Theory (Second Edition) (Prentice Hall, USA, 2001).

Received 23 September 2014

Revised 11 February 2015

Accepted 11 February 2015 